\title{
Differential effects of several retrieval cues over time: Evidence for time-dependent reorganization of memory
}

\author{
PASCALE GISQUET-VERRIER, ANNE DEKEYNE, and TATIANA ALEXINSKY \\ C.N.R.S., Gif sur Yvette, France
}

\begin{abstract}
The retention performance following partial training (15 trials) of a brightness-discrimination avoidance task has been shown to fluctuate over time, with a drop in performance $1 \mathrm{~h}$ after training (Kamin effect), a long-term spontaneous improvement (LTSI) after 3 days, and long-term spontaneous forgetting after 21 days. The purpose of this paper was to determine if these time-dependent modulations of retention performance reflect time-dependent modifications in the organization of the attributes that constitute the memory trace. We studied the relative effectiveness of several pretest cuings on retention performance when they were delivered just before a retention test occurring $1 \mathrm{~h}, 3$ days, or 21 days following initial training. Some cuing treatments were related to a particular training event (conditioned or unconditioned stimulus, experimental context). Compound cuings, composed of two or more training events, were also studied to test a possible additive effect between retrieval cues. To demonstrate time-dependent modifications in the memory trace, a differential effectiveness over time was expected for at least some retrieval cues. The results show that cuing may compensate for performance deficits $(1 \mathrm{~h}$ or 21 days), but does not further enhance performance when spontaneously improved ( 3 days). No detectable additive effects of retrieval cues were obtained. However, these results provide information that suggests a time-dependent effectiveness for some cuing treatments. In the last experiment, we investigated this possibility by studying the effects of each of these treatments when delivered after either a 1-h or a 21-day retention interval. The results confirm a time-dependent decrease in effectiveness of a pretest exposure to the CS and an increase in effectiveness over time of a pretest exposure to the experimental context or to a well-ordered sequence of events. Such differential retrievability of a partially learned episode according to both the nature of the cues and the length of the retention interval suggests a time-dependent reorganization of memory attributes.
\end{abstract}

The contention that the memory trace gradually decays as a function of time, fading and ultimately disintegrating, has been challenged on both experimental and theoretical grounds. Several authors have shown that the level of performance waxes and wanes, more or less regularly, as a function of the length of the training-to-test interval (TTI) (Cherkin, 1971; Holloway \& Wansley, 1973; Huppert \& Deutsch, 1969; Kamin, 1957; Messenger, 1971; Sanders \& Barlow, 1971). Furthermore, it is now well documented that spontaneous forgetting that occurs long after training is not due to a loss of the initial information, but rather to a retrieval failure (Deweer, Sara, \& Hars, 1980; Spear, 1973, 1978). We have recently shown the multiphasic nature of the retention function following partially learned brightness-discrimination avoidance training (Gisquet-Verrier \& Alexinsky, 1988) (Figure 1). After 15 massed training trials, retention performance deteriorated after $1 \mathrm{~h}$ (Kamin effect) and improved after 3 days (reminiscence, or long-term spontaneous improvement: LTSI). Both of these fluctuations preceded the more

Correspondence may be addressed to Pascale Gisquet-Verrier, Département de Psychophysiologie, LPN2, C.N.R.S., F91190, Gif sur Yvette, France. durable deterioration, observed 21 days following training, that corresponds to long-term forgetting.

It has become well accepted that a memory trace is multidimensional in nature and is composed of a collection of memory attributes constituting the internal representations of the various events encountered during the training phase and developing independently over time (Bower, 1967; Spear, 1978; Underwood, 1969). According to that position, it can be hypothesized that the organization of the attributes that constitute the memory trace is modified over time.

For Tulving and Watkins (1975), "traces are conceptualized as structured aggregates of trace elements and are defined as relations between the conditions and the products of retrieval" (p. 261). These authors used the retrieval cuing method to gain information about the composition of memory traces concerning word events in humans. According to the encoding-specificity principle (Tulving \& Thomson, 1973), a retrieval cue (pretest exposure to some training events) is effective to the extent that its informational content matches the informational content of the trace. The general view holds that the presentation of the cue activates the corresponding memory attribute. This reactivation may be able, under some 


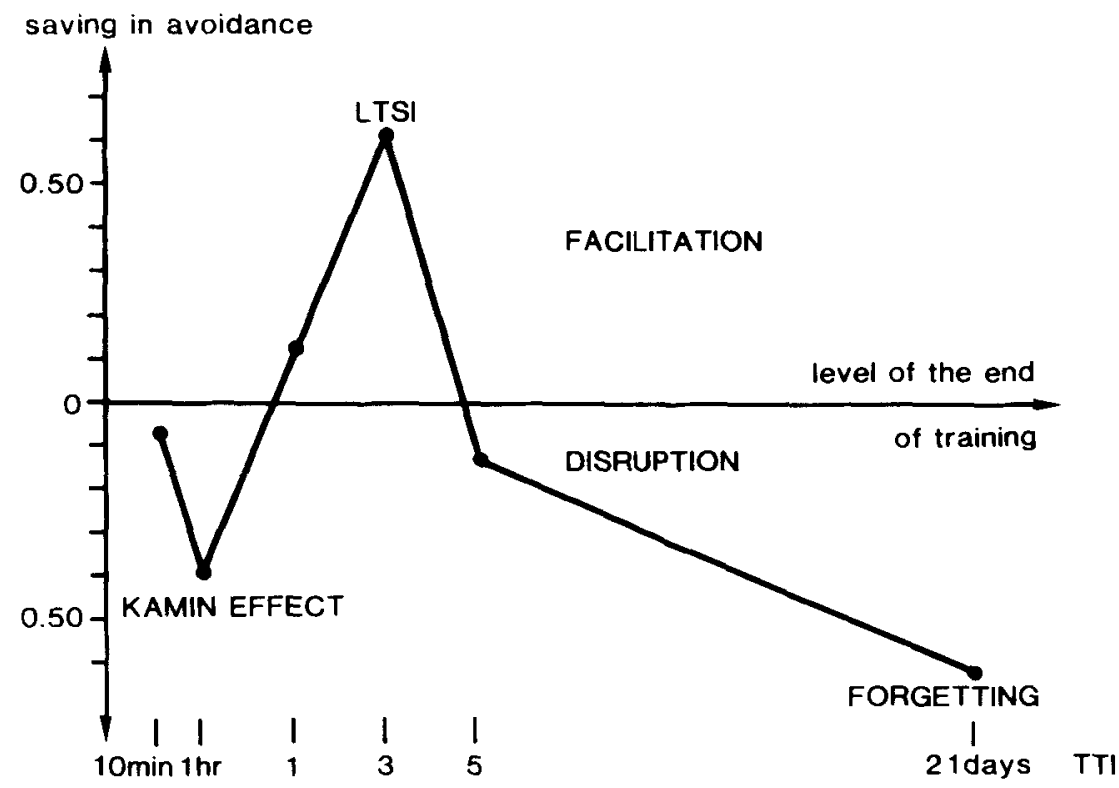

Figure 1. Mean score of savings in avoidance responses (difference between the number of avoidance responses obtained during the first five testing trials and the last five training trials) obtained for independent groups tested after various training-to-test intervals (abscissa). (Adapted from "Time-dependent fluctuations of retention performance in an aversively motivated task," by P. Gisquet-Verrier \& T. Alexinsky, 1988, Animal Learning \& Behavior, 16, 58-66).

circumstances, to trigger reactivation of other attributes linked with the initial one (Spear, 1978). This may ultimately lead the whole memory trace to pass from a passive to an active state (Lewis, 1979). According to that position, an additive hypothesis, which postulates that increasing the number of retrieval cues might facilitate the reactivation of the memory trace, has often been proposed (Spear, 1978).

In animals, retrieval cues have been shown to alleviate various retention deficits, such as experimentally induced amnesia (Koppenaal, Jagoda, \& Cruce, 1967; Miller \& Springer, 1972; Sara, 1973) or long-term forgetting (Deweer et al., 1980; Gatti, Pais, \& Weeks, 1975; Gisquet-Verrier \& Alexinsky, 1986). Typically, cuing consists of an exposure to the unconditioned stimulus (usually a negative reinforcer) in studies of experimental amnesia, or an exposure to the experimental context in studies of long-term forgetting.

If, as previously proposed, the organization of memory attributes is modified over time, the effectiveness of a particular cuing treatment on the retrieval process could depend on the memory-trace structure at the time of cuing and thus may change as a function of the length of the retention interval. However, only a few studies have concerned the relative effectiveness of several cuing treatments for a particular retention interval (Deweer, 1986; Gisquet-Verrier \& Alexinsky, 1986; Spear \& Parsons, 1976), and the time-dependent effectiveness of a samecuing treatment has been largely neglected.
The principal aim of the present experiments was to investigate the possibility of a time-dependent differential effectiveness for some cuing treatments. For that purpose, the first series of experiments was planned to study the relative effectiveness of several retrieval cues after each of the three particular retention intervals already described and corresponding to the Kamin effect (1-h TTI), LTSI (3-day TTI), and long-term forgetting (21-day TTI).

Because previous studies have shown their effectiveness in overcoming a particular case of performance disruption, retrieval cues corresponding to an exposure to separate events of the training situation, such as the conditioned stimulus (CS), the unconditioned stimulus (UCS), or the experimental context, were studied. Furthermore, to test the general hypothesis of an additive effect of cues on retrieval processes (Spear, 1978), the effects of treatments constituted by the combination of separate training events were also studied. Cuing effectiveness was assessed by comparing cued and noncued animals as well as cued and control-cued animals. This series of experiments provides information concerning the functioning of the retrieval processes during each of the three studied retention intervals as well as the cuing phenomenon and, more particularly, a possible additive effect between cues.

The second experiment was specifically addressed to test possible time-dependent cuing effectiveness. Three cuing treatments for which differential effects over time could be expected, given the results obtained in the first series of experiments, were selected. The effectiveness 
of these treatments that corresponded to an exposure to the CS, to the experimental context, or to a sequence of well-ordered training events, was studied when the cues were delivered after either a 1-h or a 21-day retention interval.

\section{Determination of the Cuing Treatments}

UCS, CS, and context. Pretest exposure to the UCS (most commonly, noncontingent footshocks) has been shown to alleviate various cases of performance deficits resulting from amnesic treatments (Miller \& Springer, 1972), spontaneous forgetting (Spear \& Parsons, 1976), and even from the Kamin effect (Klein \& Spear, 1970). The latter study is the only one showing a recovery from a performance deficit resulting from an intermediate retention interval. UCS presentation used in that study served as a model for the cue presentation of the present study. Animals in Group BGS (box + grid + shock) were placed in the cuing box located in the colony room and received five 2-sec electrical footshocks at the same intensity as the one used during training, spaced by 28 -sec intervals. Cuing ended $5 \mathrm{~min}$ prior to the retention test, allowing most of the proactive effect of the electrical shock to dissipate. Because consistent data has demonstrated the effectiveness of such an exposure, BGS treatment was expected to induce a facilitative effect on retention performance, at least when disrupted ( $1 \mathrm{~h}$ and 21 days).

The other cuing treatments replicated most of the characteristics of treatment BGS: same duration (2.5 $\mathrm{min}$ ), same number of cue presentations (five), and same cuing-to-test interval ( $5 \mathrm{~min}$ ). Figure 2 illustrates the nine cuing treatments studied.

Some studies have indicated that a pretest exposure to the CS may result in an improvement in retention performance (Gordon, Smith, \& Katz, 1979; Rohrbaugh, Riccio, \& Arthur, 1972). Furthermore, an exposure to the CS has been used successfully as a reminder treatment following experimental amnesia induced by electroconvulsive shock (ECS) (Gordon \& Mowrer, 1980), and has been shown to make the memory trace sensitive again to an ECS treatment, delivered $24 \mathrm{~h}$ after fear conditioning (Misanin, Miller, \& Lewis, 1968). More recently, it was observed that blocking and overshadowing can be prevented by a pretest exposure to the CS (Balaz, Gutsin, Cacheiro, \& Miller, 1982; Kasprow, Cacheiro, Balaz, $\&$ Miller, 1982). The CS was delivered in the same way as the UCS: animals in Group BL (box + light) were placed in the cuing box and were exposed to a series of five 2 -sec illuminations presented every $28 \mathrm{sec}$ and delivered by a $40-\mathrm{W}$ lamp, similar to the one used during training. In every one of the few studies that have established the effectiveness of a pretest exposure to the CS, the treatment was always delivered relatively soon after training (see Gisquet-Verrier \& Alexinsky, 1989). For that reason, Treatment $\mathrm{BL}$ was expected to be effective for the shortest retention intervals and no prediction was made for the longest TTI.
In our laboratory, pretest exposure to the experimental context has been repeatedly shown to be highly effective in attenuating the performance deficit resulting from spontaneous forgetting (Deweer, 1986; Deweer et al., 1980; Gisquet-Verrier \& Alexinsky, 1986). Animals in Group CONT (experimental context) were exposed to the experimental room for $2.5 \mathrm{~min}$, in a wire-mesh cage next to the Y-maze. A facilitative effect of Treatment CONT was strongly expected after 21 days and no prediction was made for the shortest retention intervals.

Control groups. Cued animals were compared not only to the noncued animals, but also to control animals.

Group B (box) was aimed at controlling the effects of the manipulation: animals in this group were simply placed in the cuing box during a 2.5 -min period. Comparison between this group and another one permitted assessment of the specific effect of a particular cue.

The other control group (Group TRIAL) constituted a reference level for retraining. Animals in this group had one complete training trial in the $\mathrm{Y}$-maze and thus were exposed to all the training events. Obviously, equivalence between Treatment TRIAL and others during which animals received multiple exposures to separate cues is difficult to establish. In fact, Treatment TRIAL was only planned to compare the effects of a cuing treatment to those of explicit retraining. Treatment TRIAL was expected to induce a facilitative effect independent of the length of the TTI.

Other cuing treatments. An assumption based upon an additivity between retrieval cues would be that the greater the number of training cues included in the cuing treatment, the greater the effectiveness of the cuing treatment. The following groups were added to test such a possibility.

Spear and Parsons (1976) suggested that, in a negatively reinforced task, exposure to the grid could act as a reactivation treatment. To test such a possibility, animals in Group BG (box + grid) were exposed to the box equipped with a grid floor identical to the floor of the Y-maze (intramaze context). This group was included to test the effects of the explicit UCS relative to the suggested UCS (by comparing the BG and BGS groups). Comparison of Group BG to Group B permits an assessment of the effectiveness of the grid by itself.

Animals in Group BGL (box + grid + light) were placed in the cuing box equipped with the grid floor and were exposed to the five illuminations given to Group BL.

Animals in Group BGSL (box + grid + shock + light) were exposed to five CS and five UCS presentations. They were placed in the cuing box with the grid floor and, every $13 \mathrm{sec}$, they received a 2 -sec electrical footshock or a 2-sec light exposure, presented in alternation.

Animals in Group SEQ (sequence of events) were exposed to the same training events as those of Group BGSL, but arranged in an ordered sequence close to the one of training: an initial 23-sec illumination period followed by a 7 -sec period of darkness, ending with a 2 -sec elec- 


\section{PRETEST CUING TREATMENTS}

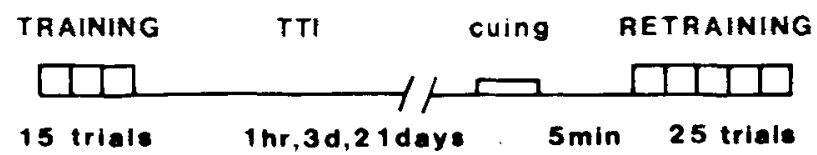

\section{COLONY ROOM}

1
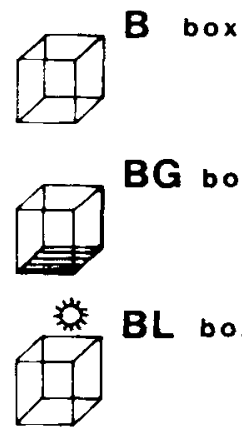

BG box-grid

\section{$2 \min 30$}

3

$B L$ box-iight

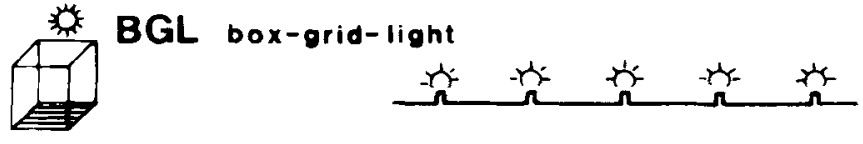

5

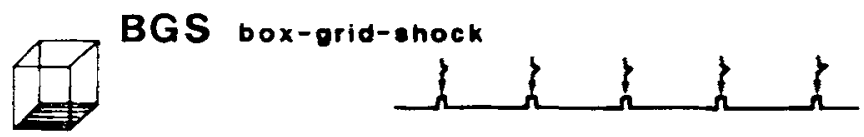

6

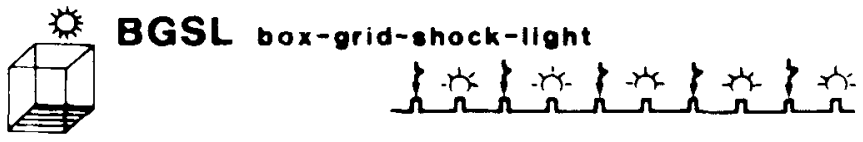

7

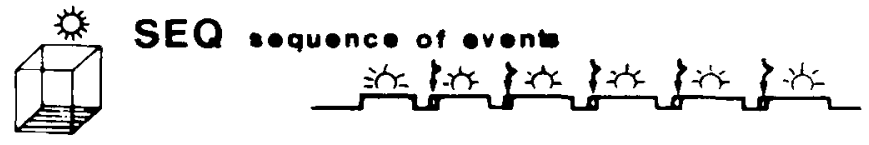

\section{EXPERIMENTAL ROOM}
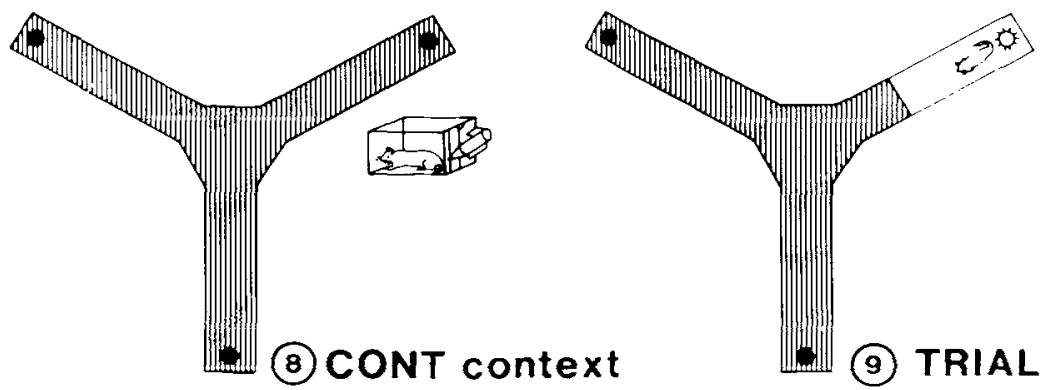

Figure 2. Experimental design. The different cuing treatments started 7.5 min before the retention test ( $2.5 \mathrm{~min}$ of cuing $+5 \mathrm{~min}$ of cuing-to-test interval), which occurred $1 \mathrm{~b}, 3$ days, or 21 days following initial training. Note that two cuing treatments were delivered in the experimental room (CONT and TRIAL). 
trical footshock. This sequence of events was repeated five times.

According to the additive hypothesis, a progressive increase in cuing effectiveness (with a possible ceiling effect) was expected among Groups $\mathrm{B} \rightarrow \mathrm{BL} \rightarrow \mathrm{BGL} \rightarrow$ BGSL $\rightarrow$ SEQ as well as among Groups $\mathrm{B} \rightarrow \mathrm{BG} \rightarrow \mathrm{BGS}$ $\rightarrow$ BGSL $\rightarrow$ SEQ.

\section{GENERAL METHOD}

\section{Subjects}

Male Sprague-Dawley rats were obtained from the Iffa-Credo rearing center at 50-57 days of age, weighing $250 \mathrm{~g}$ at their arrival in the laboratory. They were housed in pairs in wire-mesh cages and had free access to food and water throughout the experiment.

\section{Apparatus}

Training and testing were carried out in a fully automated black perspex $Y$-maze with arms $13 \mathrm{~cm}$ wide $\times 60 \mathrm{~cm}$ long $\times 38 \mathrm{~cm}$ high and a $25-\mathrm{cm}$ equilateral choice area. A 40-W lamp was located at the end of each arm. The floor consisted of a 3-mm-diameter grid, spaced $9 \mathrm{~mm}$ apart. A shock generator (Campden 521S), set to deliver scrambled shock, provided the incentive for learning. Photocells placed $1 \mathrm{~cm}$ (proximal), $11 \mathrm{~cm}$ (medial) and $30 \mathrm{~cm}$ (distal) from the entrance of each arm were connected to an Apple II microprocessor that controlled the light sequence and the length of the intertrial interval, and recorded latencies and the sequence of visited arms. The maze was housed in a darkened room adjacent to the colony room.

Cuing was delivered in either a cuing box or a wire-mesh cage that was similar to the animals' home cages. The cuing box largely differed from the experimental apparatus in both size $(23 \times 23 \times 36 \mathrm{~cm})$ and color (beige). It was covered by a removable ceiling, equipped or not with a 40-W lamp. The cuing box was placed in the colony room.

\section{Procedure}

Several days after their arrival, the rats were handled 2 at a time for $5 \mathrm{~min}$.

Pretraining. On Day 1 , the rats, in groups of 6 , were allowed to explore the entirely lighted maze for $15 \mathrm{~min}$. On Day 2, each rat was placed in the lighted maze and was allowed to explore the apparatus for $5 \mathrm{~min}$.

Training. Training began on the following day. All animals were given 15 training trials according to the following procedure. Each rat was placed in the lighted arm of the Y-maze for $20 \mathrm{sec}$. The door closing the start arm (used only on the first trial) was then opened, the light was turned off, and a second arm was lighted. Five seconds later, an electric shock was applied to the grid floor in the whole maze, except the terminal $30 \mathrm{~cm}$ of the lighted arm. Throughout the experiment, the intensity of the electrical shock was adapted for each animal just below the squeak threshold and corresponded to the minimal level for eliciting a rapid response (intensity between .3 and $.5 \mathrm{~mA}$ ). The rat had to run to the lighted arm to escape the footshock. The time elapsed from the lighting of a new goal arm until the subject crossed the proximal photobeam of the initial arm was defined as the start latency. The time elapsed until the subject crossed the distal photobeam of the new goal arm was defined as the response latency. When the animal crossed the medial photobeam of an arm, the arm was considered to have been chosen. An incorrect response was scored when the rat entered into one (or more) of the dark alleys before escaping into the lighted alley. Animals that failed to respond within $60 \mathrm{sec}$ were manually pushed into the lighted arm; a response latency of $60 \mathrm{sec}$ was then scored. The rat remained in the safe compartment for a 20 -sec period (the intertrial interval). During this period, an exit from the lighted alley led to shock. The goal alley on any given trial served as start alley for the next trial. The sequence of correct right and left turns in the $Y$-maze was determined according to the following pseudorandom schedule: L-L-R-L-R-R-L-R-R-L-R-L-L-R-R.

After completing the 15 training trials, the rats were removed from the maze and were returned to their home cages, where they remained for their TTI. To homogenize the different groups, each animal was then assigned to one of the 10 experimental conditions on the basis of its performance during the whole training phase (response latency, number of errors, and number of avoidances). Rats were discarded whenever the mean response latency obtained during the last five training trials was greater than $30 \mathrm{sec}$. Thirtynine rats out of 623 were discarded in this series of experiments.

Testing. At the end of the retention interval, the noncued animals (NC) were removed from their home cages and were carried in the experimenter's hand to the adjacent experimental room. They were placed directly into the lighted starting arm. Twenty seconds later, the starting door was opened and the animals were retrained for 25 trials in the same conditions as during initial training, with the lighted arm positioned according to the following sequence: L-R-R-R-L-L-R-L-L-L-R-L-L-R-L-R-R-R-L-L-R-R-L-R-R.

Cuing. Before the testing session, the animals in the cued condition were given one of the nine pretest cuing treatments illustrated in Figure 2. Cuing lasted $2.5 \mathrm{~min}$ (except for Treatment TRIAL) and was delivered either in the cuing box placed in the colony room (Treatment 1 to Treatment 7) or in the experimental room (Treatments 8 and 9).

At the end of the treatments, all cued animals were returned to their home cages for $5 \mathrm{~min}$. The testing procedure was identical to that of noncued animals.

\section{Behavioral Measures}

Start latencies, response latencies, and sequences of visited arms were recorded for each training and test trial. A response latency of less than $5 \mathrm{sec}$ permitted avoidance of the shock and was scored 2 . A start latency below 5 sec corresponded to an exit from the initial arm, emitted during the CS-UCS interval. Such a response was considered as an attempt to avoid the shock and was scored 1. The response latencies, the number of errors, and the avoidance score were pooled in blocks of five trials for both training and testing. To normalize the measures, logarithmic transform was applied to the response latencies before statistical analyses. The response latencies, the number of errors, and the avoidance score were used to assess a learning process during initial training. Savings in avoidance, expressed as the difference between the mean score of the first five testing trials and that of the last five training trials, were taken as a measure of retention. This measure has been shown to be the most appropriate way to express retention performance in this particular task (for more detailed information, see GisquetVerrier \& Alexinsky, 1988).

\section{Statistical Analyses}

Statistical analyses for each experiment involved within-group comparisons, which compared performance during the first five testing trials and the last five training trials, and paired comparisons performed on savings in avoidance.

Each of the cued groups was compared to the noncued group as well as to the control groups (Group B and Group TRIAL).

Comparisons between two groups of animals differing only by the exposure to an additional training event $(B \rightarrow B G \rightarrow B G S \rightarrow B G S L$ $\rightarrow \mathrm{SEQ}$ and $\mathrm{B} \rightarrow \mathrm{BL} \rightarrow \mathrm{BGL} \rightarrow \mathrm{BGSL} \rightarrow \mathrm{SEQ}$ ) were performed to test a possible additive effect between cues. 


\section{FIRST EXPERIMENTS \\ 1-HOUR, 3-DAY, AND 21-DAY TTI}

\section{Method}

\section{Initial Training}

In each experiment, some animals were discarded at the end of training because of excessive response latencies: 9 for the 1-h TTI, 12 for the 3-day TTI, and 12 for the 21-day TTI.

An analysis of variance (block $\times$ group) was performed on the three blocks of the training period and among the 10 groups that subsequently differed in pretest conditions. In each experiment, this analysis revealed a significant effect of repetition on response latencies, errors, and avoidances ( $p<.001$ in every case). There was no significant difference among groups nor significant interaction between groups and blocks, regardless of the considered variable. These results indicate an ongoing acquisition process and an unbiased distribution of the animals to the different groups.

\section{Retention}

1-h TTI. Each of the 166 rats that served as subjects in this experiment was assigned to one of the 10 following groups: Group NC $(n=18)$, Group B $(n=17)$, Group BG $(n=16)$, Group BL $(n$ $=17)$, Group BGS $(n=16)$, Group BGL $(n=17)$, Group BGSL $(n=17)$, Group SEQ $(n=17)$, Group CONT $(n=16)$, and Group TRIAL $(n=15)$.

3-day TTI. As in the first experiment, the 158 animals of this experiment were assigned to one of 10 groups: Group NC
( $n=17)$, Group B $(n=17)$, Group BG $(n=16)$, Group BL $(n=17)$, Group BGS $(n=15)$, Group BGL $(n=15)$, Group BGSL $(n=15)$, Group SEQ $(n=16)$, Group CONT $(n=16)$, and Group TRIAL $(n=16)$. Mean savings in avoidance obtained for the different groups are represented in Figure 4.

21-day TTI. The 173 animals of this experiment were also assigned to one of the 10 following groups: Group NC $(n=16)$, Group B $(n=18)$, Group BG $(n=18)$, Group BL $(n=18)$, Group BGS $(n=18)$, Group BGL $(n=17)$, Group BGSL $(n=$ 17), Group SEQ $(n=17)$, Group CONT $(n=17)$, and Group TRIAL $(n=18)$. Mean savings in avoidance obtained for the different groups are represented in Figure 5.

\section{Results and Discussion}

\section{1-h TTI}

Mean savings in avoidance obtained for the different groups of animals are illustrated in Figure 3.

Within-group comparisons showed an impairment of the avoidance performance of the noncued animals (Group NC) between the last block of training trials and the first block of testing trials $[F(1,17)=4.51, p=.046]$. On the other hand, the animals of Group BL exhibited a significant improvement in their avoidance performance $[F(1,16)=$ $21.76, p<.001]$. No other comparison led to a significant difference.

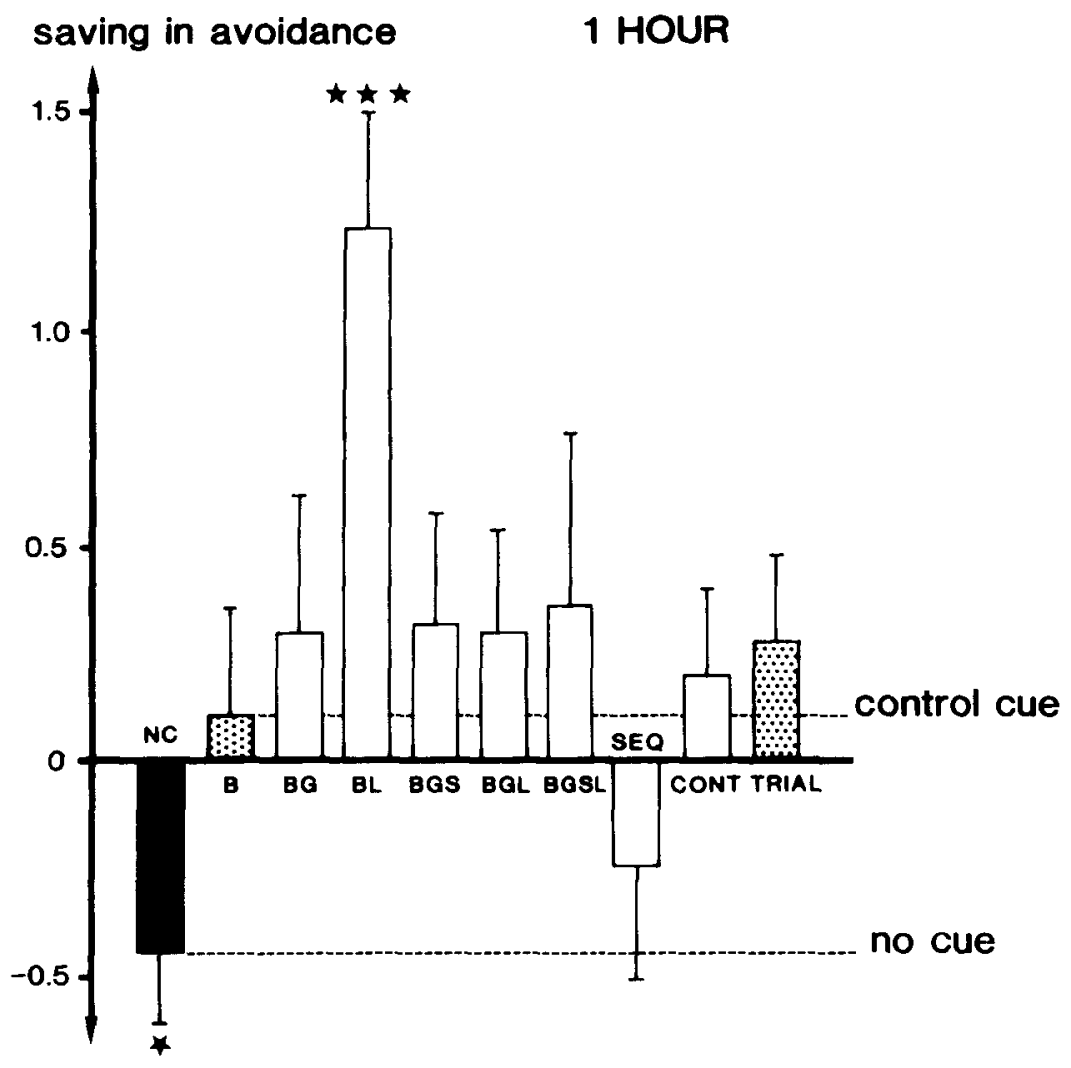

Figure 3. Mean score $( \pm S E M)$ of savings in avoidance at the 1-h TTI (same conventions as for Figure 1). Note the disruption in the avoidance score for the noncued group (NC) relative to the end of training (abscissa), and the large improvement in performance induced by Treatment BL. (Within-group comparisons: $* .025<p<.05$; $* * p<.001$.) Black column $=$ noncued group; dotted columns $=$ control-cued groups $(B$ and TRIAL). 
Paired comparisons showed that the noncued animals (Group NC) did not significantly differ from the animals in Control Group $B[F(1,33)=1.64]$, but they exhibited significantly less savings in avoidance than did the reference Control Group TRIAL $[F(1,31)=5.67, p=.002]$. There was no significant difference between the two control groups B and TRIAL $(F<1)$.

The animals in Group BL exhibited more savings in avoidance than did the noncued animals $[F(1,33)=25.94$, $p<.001]$ and the animals in the two control groups, $\mathrm{B}$ and TRIAL $[F(1,32)=8.56, p=.006$, and $F(1,30)$ $=8.01, p=.008$, respectively]. In addition, Group BL showed better retention performance than any of the remaining groups $(p=.008<p<.027)$.

The animals in Groups BG and BGS had significantly more savings in avoidance than those of the noncued group $[F(1,32)=4.21, p=.046$, and $F(1,32)=4.82, p=$ .033 , respectively] but did not significantly differ from those of the two control groups, B and TRIAL $(F<1$ in each case). No difference between Group BGS and Group BG could be detected $(F<1)$.

The animals in Groups BGL and CONT performed significantly better than the noncued animals $[F(1,33)=$ $5.22, p=.027$, and $F(1,32)=4.35, p=.042$, respectively], but there was no significant difference between each of these groups and the two control groups, B and TRIAL $(F<1)$.

The animals in Groups BGSL and SEQ did not significantly differ from the noncued animals $[F(1,33)=1.83$ and $F<1$, respectively]. Nor did they differ significantly from the control groups, B and TRIAL (all $F \mathrm{~s}<1$ ).

As indicated in Figure 3, the results obtained after the 1-h TTI do not indicate any progressive increase in cuing effectiveness as a function of the number of presented cues (see $\mathrm{B} \rightarrow \mathrm{BG} \rightarrow \mathrm{BGS} \rightarrow \mathrm{BGSL} \rightarrow \mathrm{SEQ}$ and $\mathrm{B} \rightarrow \mathrm{BL} \rightarrow$ BGL $\rightarrow$ BGSL $\rightarrow$ SEQ). A dramatic facilitative effect on retention performance was obtained with additional exposure to the light delivered in the cuing box $(\mathrm{B} \rightarrow \mathrm{BL})$. However, this effect was not obtained when the animals were placed in the cuing box equipped with a grid (BG $\rightarrow$ BGL) or when the light was delivered in addition to the UCS (BGS $\rightarrow$ BGSL). On the other hand, adding the grid to the cuing box significantly reduced the cuing effectiveness of the light alone: $\mathrm{BL} \rightarrow \operatorname{BGL}[F(1,32)=$ 6.97, $p=.012$ ].

After a 1-h TTI, most of the cuing treatments induced a significant enhancement of retention performance, relative to the performance of noncued animals (Treatments BG, BL, BGS, and CONT). However, exposure to the CS (Group BL) was significantly more effective than any of the remaining cuing treatments, including the reference control, TRIAL. These results confirm those obtained by Klein and Spear (1970) in showing that a pretest exposure to the UCS (Group BGS) abolished the performance deficit normally obtained in noncued animals. Spear (1978) held that this treatment acts on the internal state of the animals, since the same results were obtained by a pretest stimulation of the hypothalamus that mimicked the physiological consequences of electrical footshocks (Klein, 1972). For Spear, at the time of testing, the internal context becomes more like that which prevailed during learning, and this, in turn, increases the effectiveness of the memory retrieval. However, the determining factor of the cuing effectiveness at the 1-h retention interval does not seem to be an exposure to the UCS, for three principal reasons: (1) an exposure to the grid alone was as effective as an exposure to the grid plus the footshocks; (2) the most effective treatment (BL) did not include any electrical footshock; and (3) another treatment during which UCS was presented (SEQ) did not induce a similar facilitative effect.

It must be pointed out that the poor performance of the noncued rats was very transient since no difference between cued and noncued animals was noticeable after the first block of testing trials, whereas avoidance performance significantly increased thereafter. This result is not in line with an explanation of the Kamin effect postulating a physiologically based inhibition that would prevent the animals from performing adequately in an aversive situation (Anisman, 1975; Brush, 1971), for which a longer lasting effect would be expected. Transience and alleviation by pretest cuing of the performance deficit are more compatible with an explanation of the Kamin effect in terms of retrieval failure.

These results indicate that the 1-h performance deficit can be overcome by a wide variety of cuing treatments. However, exposure to the CS appears to be particularly effective in that it did not only alleviate the drop of performance, but also induced a large improvement in retention performance.

\section{3-Day TTI}

Mean savings in avoidance obtained for the different groups are represented in Figure 4.

Within-group comparisons indicated an improvement in the avoidance performance for the noncued animals (Group NC) between the end of training and the beginning of testing $[F(1,16)=4.81, p=.041]$. Group BG exhibited a significant improvement in performance $[F(1,15)=8.59, p=.01)$. Improvement in performance fell just short of significance for Group TRIAL $[F(1,15)$ $=4.23, p=.055]$. No other within-group comparisons led to a significant difference.

Paired comparisons showed that the noncued animals did not significantly differ from either of the control groups, B and TRIAL $(F \mathrm{~s}<1)$, and no difference between the two control groups could be detected $(F<1)$.

The animals of Groups BGS, BL, and CONT, as well as those of Groups BG, BGSL, and SEQ did not differ significantly either from the noncued animals or from either of the two control groups, B and TRIAL. There was no difference between the avoidance score of Group BG and Group BGS.

The animals of Group BGL exhibited significantly less savings in avoidance than did the noncued animals $[F(1,30)$ $=7.14, p=.011]$, Control Group B $[F(1,30)=5.31$, 


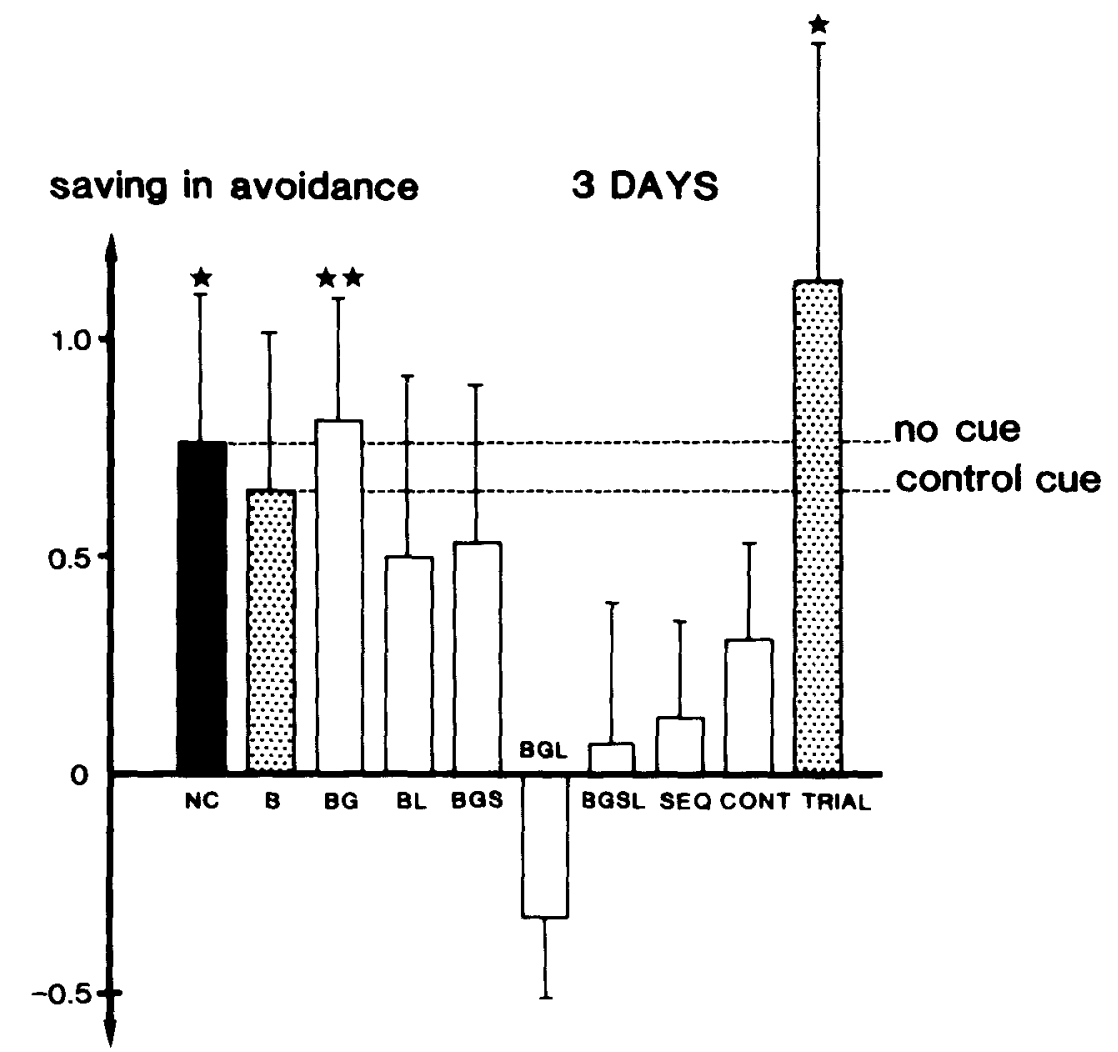

Figure 4. Mean score $( \pm S E M)$ of savings in avoidence at the 3-day TTI. Note the spontaneous increase in performance for the noncued animals (NC) relative to the end of training. Note also that none of the cuing treatments further enhanced retention performance relative to Group NC. (Within-group comparisons: *.025 $<p<.05 ; *$ *001 $<p<.025$.)

$p=.026]$, and Reference Control Group TRIAL $[F(1,29)$

$=6.04, p=.019]$.

As indicated in Figure 4, these results did not indicate any progressive increase in cuing effectiveness related to the number of the training events presented during $\mathrm{cu}$ ing. On the contrary, the effectiveness of Treatment BG was significantly decreased by the additional exposure to the light, as assessed by the comparison between Group BG and Group BGL $[F(1,29)=11.43, p=.002]$.

After a 3-day retention interval, the performance of the noncued animals was spontaneously enhanced, relative to that at the end of training ("spontaneous" means that nothing but time was an intervening factor). In these conditions, none of the cuing treatments further enhanced retention performance. This lack of facilitation was not due to a ceiling effect because the number of avoidances at the beginning of testing was far from that reached by the noncued animals at the end of retraining.

It has already been proposed that cuing treatment can only exert a facilitative effect on retention performance insofar as memory is not easily accessible (Gordon \& Mowrer, 1980; Gordon et al., 1979; Spear, Hamberg, \& Bryan, 1980; Spear \& Parsons, 1976). Our results, which indicate that none of the studied cuing treatments was able to enhance retention performance when delivered after a 3-day TTI, support the notion that LTSI corresponds to a period during which retrieval processes work at their optimal level. Such a position implies that retrieval processes might be depressed before LTSI.

In fact, spontaneous improvement of performance was preserved only following Treatments BG and TRIAL, for which there was complete matching between training and cuing information. Other cuings, in which training events were presented in a way somehow different from that used during training, tended to have a disruptive effect. This effect is particularly clear for Treatment BGL.

\section{1-Day TTI}

Mean savings in avoidance obtained in the different groups are represented in Figure 5.

Within-group comparisons indicated that following a 21-day TTI, the noncued animals (Group NC) exhibited a significant decrease in avoidance performance, relative to the end of training $[F(1,15)=4.32, p=.05]$. On the other hand, the avoidance performance of the animals in Group BG was significantly improved $[F(1,16)=6.37$, $p=.021]$, as was the performance of the rats in Group SEQ $[F(1,16)=19.45, p<.001]$, Group CONT 


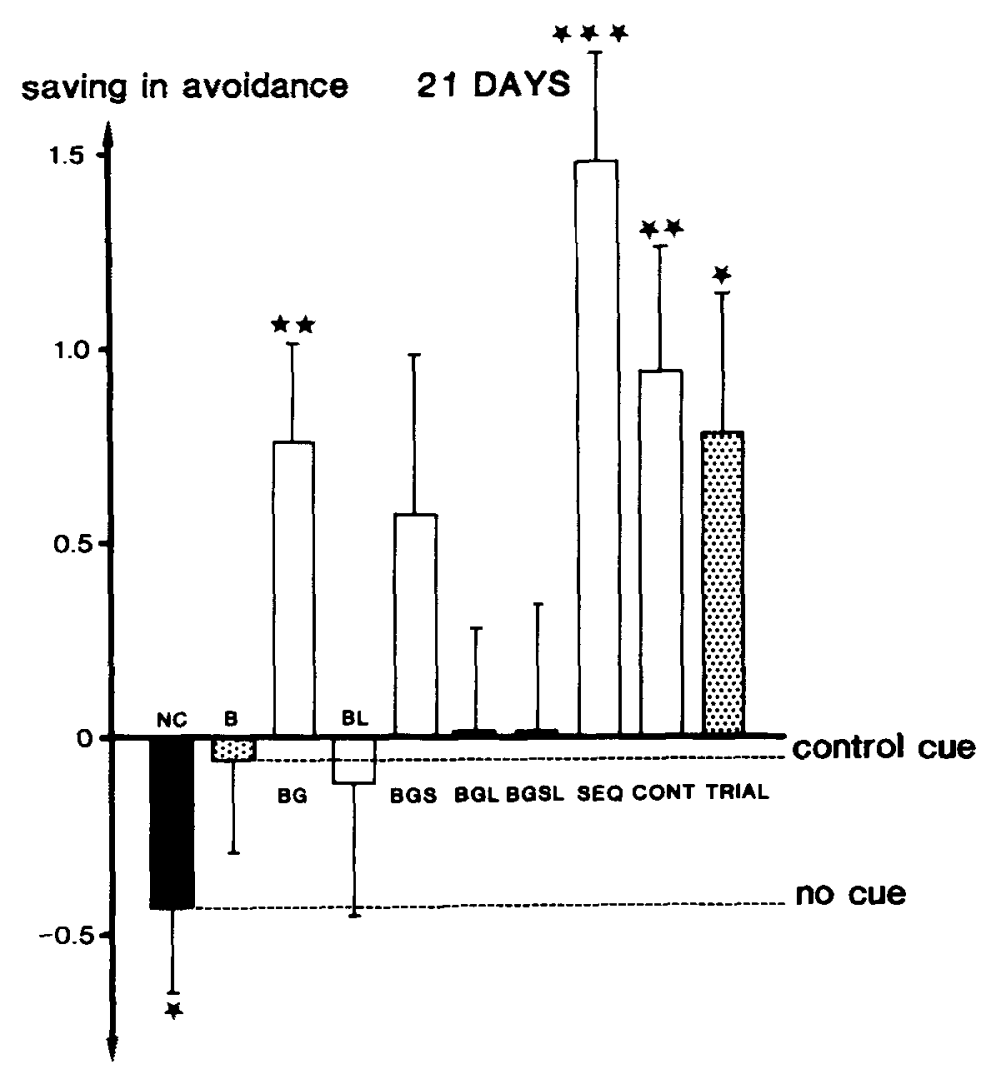

Figure 5. Mean score $( \pm S E M)$ of savings in avoidance at the 21-day TTI. Note the decrease in performance for the noncued group (NC) and the significant increase in performance following Treatments BG, SEQ, CONT, and TRIAL, relative to the end of training. (Within-group comparisons: $\left.* .025<p<.05 ; * * .001<p<.01 ;{ }^{* * *} p<.001.\right)$

$[F(1,16)=8.33, p=.010]$, and Group TRIAL $[F(1,17)$ $=4.73, p=.041]$. None of the other comparisons led to a significant difference.

Paired comparisons indicated that the noncued animals (Group NC) did not perform differently from the animals in Control Group B but exhibited significantly less savings in avoidance than did Reference Control Group TRIAL $[F(1,32)=7.84, p=.008]$. The difference between the two control groups fell just short of significance $[F(1,34)=3.66, p=.061]$.

The animals in Groups BG and BGS had significantly more savings in avoidance than did the noncued animals $[F(1,31)=10.08, p=.003$, and $F(1,32)=6.06, p=$ .018 , respectively]. The difference between Group BG and Group $B$ fell just short of significance $[F(1,33)=$ $3.87, p=.054$ ], and no difference between Group BG and Group TRIAL was obtained $(F<1)$. Group BGS did not differ from either of the two control groups $(F \mathrm{~s}<1)$.

The animals in Group BL did not perform differently either from the noncued animals or from the animals of the control groups, $\mathrm{B}(F<1)$ and TRIAL $[F(1,34)=$ $3.23, p=.078$ ].

The animals in Group CONT exhibited a larger savings score than both the noncued animals $[F(1,31)=$
$11.88, p=.002]$ and the animals in Control Group B $[F(1,33)=5.99, p=.018]$. There was no difference between Group CONT and Group TRIAL $(F<1)$.

The animals in Group SEQ exhibited more savings in avoidance than did the noncued animals $[F(1,31)=22.07$, $p<.001]$ and the animals in Control Group B $[F(1,33)$ $=13.66, p=.001]$. There was no significant difference between Group SEQ and Group TRIAL $[F(1,33)=1.99]$.

The animals in Groups BGL and BGSL exhibited no significant difference in performance relative to the noncued animals $[F(1,31)=1.53$ and $F(1,31)=1.17$, respectively]. Nor did they differ significantly from the control groups, $B(F \mathrm{~S}<1)$ and TRIAL $[F<1$ and $F(1,33)=2.97 ; p=.09$, respectively].

As indicated in Figure 5, these results did not show any consistent increase in cuing effectiveness related to the number of presented cues $(\mathrm{B} \rightarrow \mathrm{BL} \rightarrow \mathrm{BGL} \rightarrow \mathrm{BGSL} \rightarrow$ $\mathrm{SEQ}$ or $\mathrm{B} \rightarrow \mathrm{BG} \rightarrow \mathrm{BGS} \rightarrow \mathrm{BGSL} \rightarrow \mathrm{SEQ}$ ). The significant difference between Group BGSL and Group SEQ $[F(1,32)=9.77, p=.004]$ suggests that at this TTI, time relationships between the CS and the UCS are of critical importance for retrieving the initial memory.

It should be noted that the groups exposed to the light as a separate event during cuing (Groups BL, BGL, and BGSL) never significantly differed from the noncued 
group. Furthermore, adding the light in the cuing box equipped with the grid reduced the cuing effectiveness of the grid itself, but this difference did not reach an acceptable level of significance $[F(1,32)=3.01, p=.08]$.

After a 21-day TTI, the retention performance of the noncued animals was depressed, relative to the end of training. All of the cuing treatments tended to alleviate, to varying degrees, the forgetting observed in the noncued animals. This result confirms that long-term forgetting is not due to a memory loss, but to a failure to adequately retrieve the initial information (Deweer et al., 1980; Gisquet-Verrier \& Alexinsky, 1986; Miller, Kasprow, \& Schachtman, 1986; Spear, 1978).

After 21 days, the effective cuing treatments either were related to the UCS (BG, BGS) or were composed of complex information, such as the experimental context or a sequence of events. Most of them further induced an improvement in retention performance relative to the end of training (Treatments BG, SEQ, and CONT), which suggests that effective cuings restore the improvement of performance normally reached during LTSI.

\section{General Discussion of First Experiments}

This series of experiments confirms our previous results (Gisquet-Verrier \& Alexinsky, 1988) in showing that after partial avoidance training in a brightness discrimination, retention performance was disrupted after $1 \mathrm{~h}$ (Kamin effect), improved after 3 days (LTSI), and was depressed after 21 days (long-term forgetting).

An ancillary interest in this study was to test a possible additive effect between cues delivered during pretest cuing. For Spear (1978), "the elicitation and retrieval of the target attribute of a memory depend on the arousal of a sufficient number or kind of the remaining attributes of that memory" (p. 418). According to this position, the larger the number of training events during cuing, the larger the reactivation of the memory trace. The demonstration that an exposure to the training apparatus enhances test performance when an additional presentation of the UCS is provided (DeVietti \& Hopfer, 1974; Silvestri, Rohrbaugh, \& Riccio, 1970) supports this view. In the same way, Deweer (1986) showed that after a 21-day retention interval, exposure to the training context followed by access to the specific reinforcer (food) was effective in alleviating spontaneous forgetting when delivered $1 \mathrm{~h}$ before testing, whereas each treatment alone was not.

None of the three present experiments demonstrated any additive effect between cues. It appears that the "kind" more than the "number" of presented cues determines cuing effectiveness. Contrary to what was expected, it was found that, in some cases, the addition of a supplementary training feature dramatically reduced the effectiveness of a cuing treatment $(\mathrm{BL} \rightarrow \mathrm{BGL}$ after $1 \mathrm{~h}, \mathrm{BG} \rightarrow$ BGL after 3 or 21 days). The two features "light" and "grid" seem to have been in conflict. In circumstances in which one of them was effective, adding the other significantly reduced the initial effect. These findings can be accounted for by hypothesizing that presentation of the grid preferentially reactivated parts of the memory trace dealing with escape or avoidance responses, whereas presentation of the light reactivated traces related to immobility and safety. Such combined reactivations of partial traces that evoke opposite behavioral responses might interfere, and thus might depress rather than enhance retention performance. Such a hypothesis is supported by the results obtained by Spear and Parsons (1976) showing that additional presentation of the CS during exposure to the training apparatus decreases the cuing effectiveness of the latter treatment.

Taken together, our data argue against a simple additivity rule in the cuing phenomenon. However, the fact that long after training complex and global cues such as CONT and SEQ are the most effective may suggest that some additivity between cues could exist, but in a more subtle way than in a simple additivity rule.

Incidentally, it should be noted that an improvement in performance relative to the end of training might occur under various circumstances. Such an improvement was obtained after a 3-day retention interval, without any prior cuing, after a 1-h retention interval, following a pretest exposure to the CS, and after a 21-day retention interval, subsequent to different cuing treatments (BG, CONT, and SEQ). This suggests that the performance reached at the end of training does not truly reflect what has really been learned during initial acquisition. According to this position, one may consider that during training and shortly thereafter (i.e., before LTSI), performance is depressed by some negative factors, the nature of which remains to be specified. Additional experiments are needed to confirm such a possibility, which has already been taken into consideration (Miller et al., 1986).

An overview of these results could help to determine the nature of the cuing treatments for which time-dependent effectiveness could be expected, despite the fact that they were obtained in three separate experiments.

Treatment $B$ is considered as the basic control treatment. The animals cued with Treatment B were simply handled and placed in the cuing box with no specific information otherwise related to training. The animals in Group B did not exhibit performance disruption relative to the end of training but never significantly differed from the noncued animals. The slight facilitative effect of Control Treatment B can be explained in different but not mutually exclusive ways. For instance, handling and placement in a neutral place can reactivate a part of the initial memory, reduce the animals' subsequent emotivity, and/or focus attentional processes at the time of testing.

Treatment TRIAL, which corresponded to a full training trial given just before the retention test, is considered as reference control treatment during which all of the training events as well as their relationships are provided. In this treatment, new learning was deliberately induced, which probably summated with that of original conditioning. As expected, Treatment TRIAL did facilitate the otherwise depressed performance of noncued animals 
tested $1 \mathrm{~h}$ or 21 days after training, and preserved the spontaneous improvement that occurred after 3 days. After either the 1-h or the 21-day retention interval, most of the effective cuing treatments induced an improvement of performance similar to that induced by Treatment TRIAL. However, after the 1-h TTI, the animals cued with a pretest exposure to the CS, which corresponded to an extinction paradigm, exhibited a larger improvement in performance than did the animals submitted to a complete retraining trial. This result demonstrates that, contrary to what has been postulated by some authors (Gold \& King, 1974), cuing treatments might affect retention performance in some way other than by providing a new learning opportunity.

Treatment BGS, which corresponded to an exposure to the grid floor plus the footshock, compensated for the performance disruption obtained in the noncued animals after either $1 \mathrm{~h}$ or 21 days. These results confirm that a pretest exposure to the UCS can compensate for a performance deficit resulting from an intermediate retention interval (Kamin effect; see Klein \& Spear, 1970) and from long-term forgetting (Spear \& Parsons, 1976).

Treatment BG appears to be as effective as Treatment BGS in alleviating the performance deficit, which suggests that, in our conditions, training cues highly related to the UCS were as effective as the UCS itself on the retrieval processes.

Treatment BL, which corresponded to five exposures to the CS, dramatically enhanced retention performance when delivered after a 1-h TTI. This treatment not only compensated for the 1-h performance deficit but also induced a large facilitation in performance, relative to the end of training. Such an improvement was larger than that obtained for any of the remaining groups, including the group given a complete retraining trial as the cuing treatment. Following a 3- or 21-day retention interval, the animals exposed to Treatment BL did not differ from the noncued animals. In all, these results suggest a decrease in the cuing effectiveness over time of a pretest exposure to the CS.

Treatment CONT, which included exposure to the experimental context, enhanced performance when presented either $1 \mathrm{~h}$ or 21 days after training. However, the animals in Group CONT exhibited better retention performance than did the animals in Group B for only the longest TTI. These results confirm that an exposure to the experimental context is particularly effective in reducing the performance deficit resulting from long-term forgetting (Deweer, 1986; Deweer et al., 1980; see also Gatti et al., 1975). They further suggest a time-dependent increase of effectiveness for Treatment CONT.

Treatment SEQ, which included five successive presentations of the well-ordered sequence of the training events, had no effect when delivered $1 \mathrm{~h}$ after training. After 3 days, Treatment SEQ interfered with the spontaneous enhancement of performance normally observed for noncued animals. Yet, when presented after 21 days, Treatment SEQ induced a large enhancement of retention performance. The animals in this group not only performed better than the corresponding noncued animals, but also performed better than those of Control Group B, attesting to the specificity of the information provided by this cue. These results suggest a time-dependent increase in effectiveness for Treatment SEQ.

\section{SECOND EXPERIMENT}

The results obtained in the first experiments suggest a differential effectiveness of some cuing treatments as a function of the length of the retention interval. The present experiment was designed to verify a possible decrease over time in the effectiveness of a pretest exposure to the CS (Treatment BL) and an increase over time in the effectiveness of a pretest exposure to the experimental context (Treatment CONT) and for a well-ordered sequence of training events (Treatment SEQ). To that aim, each of these cuing treatments was delivered after either a 1-h or a 21-day retention interval. Four experimental conditions (NC, BL, SEQ, and CONT) were tested after each of two TTIs ( $1 \mathrm{~h}$ and 21 days), according to a $4 \times 2$ factorial design. Of the 126 animals originally trained, 6 were discarded because of their poor training performance. The 120 remaining animals were assigned to one of the eight groups $(n=15)$.

\section{Results and Discussion}

\section{Initial Training}

As in the preceding experiments, a significant improvement in performance was observed across blocks during training for each of the measures studied, with no significant difference between groups.

\section{Retention}

1-h TTI. Savings in avoidance obtained for each group are illustrated in Figure 6.

Within-group comparisons indicated that the animals exposed to the CS before a 1-h delayed-retention test exhibited an improvement in their avoidance score, relative to the end of training $[F(1,14)=22.88, p<.001]$. In contrast to the preceding experiment, the decrease in avoidance exhibited by the noncued animals was not statistically reliable. None of the other groups demonstrated any significant difference.

Paired comparisons showed that the animals in Group BL exhibited greater savings than did the noncued animals $[F(1,28)=17.70, p<.001]$ and those in the two other cued groups, Group CONT $[F(1,28)=5.06$, $p=.03]$ and Group SEQ $[F(1,28)=9.97, p=.004]$. As in the first experiment, the animals of Group CONT exhibited significantly more savings in avoidance than did the animals of Group NC $[F(1,28)=4.24, p=.046]$. The animals of Group SEQ did not significantly differ from the noncued animals $[F(1,28)=2.05]$.

21-day TTI. Within-group comparisons indicated a significant decrease in avoidance performance for the noncued animals, relative to the end of training $[F(1,14)=$ $4.83, p=.043]$. Conversely, there was a significant im- 


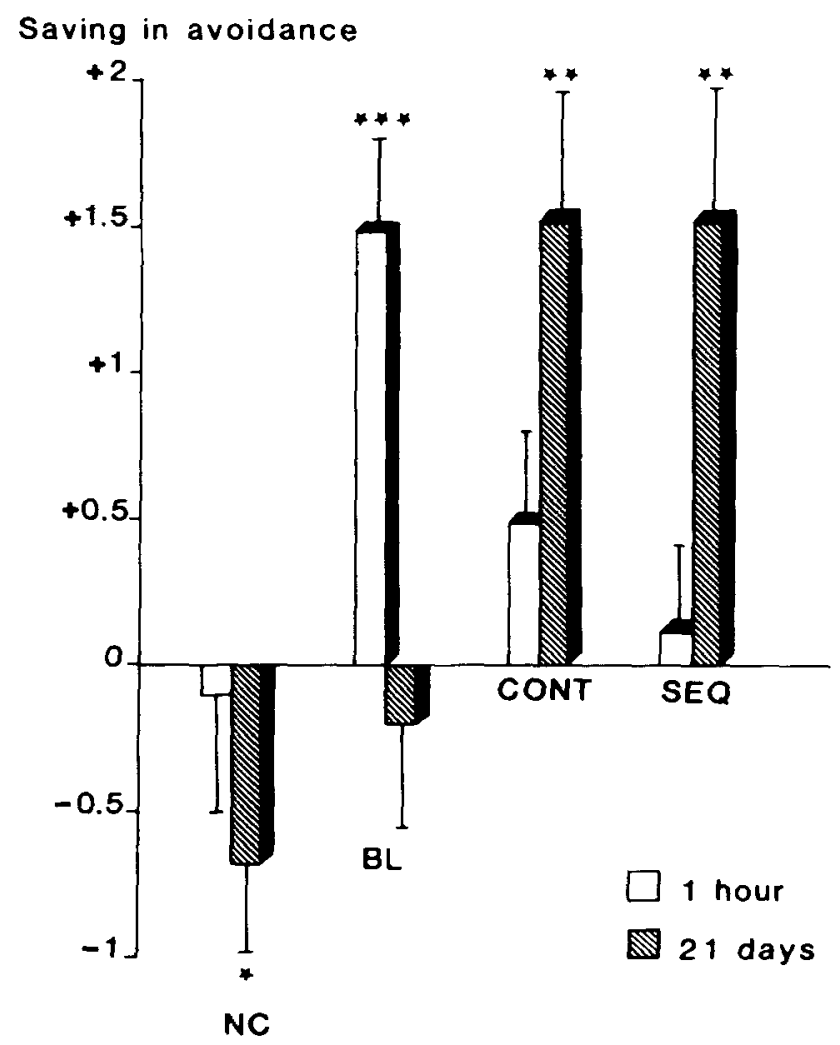

Figure 6. Mean score ( $+S E M)$ of savings in avoidance obtained after $1 \mathrm{~h}$ (white bars) or 21 days (hatched bars). Note that although there was no difference between the two noncued groups (NC), large differences were obtained following Treatments BL, CONT, and SEQ. (Within-group comparisons: $* .025<p<.05$; **.001< $p<.025 ; * * * p<.001$.)

provement of performance for the animals in Group CONT $[F(1,14)=10.34, p=.006]$ and in Group SEQ $[F(1,14)=10.67, p=.005]$.

Paired analyses indicated that the animals in Group CONT and Group SEQ exhibited significantly better retention performance than did those in the noncued group $[F(1,28)=15.16, p<.001$, and $F(1,28)=17.81$, $p<.001$, respectively]. In addition, Treatments CONT and SEQ induced greater savings in avoidance than did Treatment BL $[F(1,28)=8.29, p=.007$, and $F(1,28)$ $=9.54, p=.004$, respectively]. The animals in Group BL did not significantly differ from the noncued animals $(F<1)$.

Comparison of 1-h vs. 21-day TTIs. A $4 \times 2$ (testing conditions $\times$ length of TTI) analysis of variance performed on the savings in avoidance indicated a significant effect of testing condition $[F(3,112)=8.06$, $p<.001]$, no effect of TTI $(F \mathrm{~s}<1)$ and, most importantly for the purpose of this experiment, a significant interaction between length of TTI and testing condition $[F(3,112)=7.36, p<.001]$.

As indicated in Figure 6, the performance of the noncued animals (Group NC) was not significantly modified between $1 \mathrm{~h}$ and 21 days $(F<1)$. Cued animals, however, exhibited performance that significantly decreased between $1 \mathrm{~h}$ and 21 days for Group BL $[F(1,28)$ $=12.12, p<.001]$, and significantly increased for Group CONT $[F(1,28)=4.39, p=.042]$ and Group SEQ $[F(1,28)=7.42, p<.025]$.

The results of this experiment attest to the reliability of the results previously obtained in the first series of experiments. The present results confirm that the animals exposed to the CS before a 1-h delayed-retention test exhibited strongly enhanced retention performance. Pretest exposure to the CS has already been shown to be effective when delivered relatively soon after training (Balaz et al., 1982; Gordon et al., 1979; Kasprow et al., 1982; Rohrbaugh \& Riccio, 1970; Rohrbaugh et al., 1972; see also Gisquet-Verrier \& Alexinsky, 1989). These results further confirm that a similar facilitative effect was obtained after a pretest exposure to the experimental context or to a sequence of training events, following a 21-day retention interval.

Furthermore, this experiment demonstrated the timedependent differential effectiveness of the three selected cuing treatments. Cuing effectiveness of a pretest exposure to the CS significantly decreased over time. Such a loss of effectiveness in promoting the reactivation of the initial memory, long after training, could be due to a memory loss for specific attributes of the CS that has been demonstrated by several authors (Hendersen, Patterson, \& Jackson, 1980; Thomas \& Riccio, 1979; for more details, see Gisquet-Verrier \& Alexinsky, 1989). On the other hand, these results demonstrate that a pretest exposure to the experimental context improved retention performance after both retention intervals studied. However, the cuing effectiveness of this treatment significantly increased over time. These results lead to a paradox: How can we explain that experimental context gains effectiveness in the control of the response over time when it has already been demonstrated that a change in context between training and testing has a less and less disruptive effect on retention performance over time (Gisquet-Verrier \& Alexinsky, 1986)? These results further demonstrate a significant increase of the effectiveness of a pretest exposure to a sequence of well-ordered training events. As a matter of fact, Treatment SEQ could be considered as pseudotraining trials differing from actual training trials in that the animals had no opportunity to give the response and the events were delivered in another context. Change in context has already been shown to produce a deleterious effect shortly after training and a lesser effect long afterward (see Gisquet-Verrier \& Alexinsky, 1986). It could thus be proposed that training information delivered in a different context is unlikely to be considered by the animal as being related to the training situation, at least when presented shortly after initial training, whereas the two environments become more interchangeable over time (see also MacArdy, Kasenow, Hopkins, \& Riccio, 1987).

\section{CONCLUSION}

These experiments indicate that the use of pretest cues as probes for gaining information about memory-trace or- 
ganization, which has been effective for memory traces concerning word events in humans (Tulving \& Watkins, 1975), appears to be as effective for a memory trace concerning a discrimination-avoidance task in animals.

The results obtained in these experiments confirm the notion that a failure of retrieval processes is implicated in both studied cases of performance disruption that correspond to the Kamin effect and in long-term forgetting. In addition, the present results constitute experimental support for the multidimensional concept of the memory trace since, for each instance of performance decrement, reactivation of the memory trace can be obtained following exposure to several different cuing treatments. Conversely, during LTSI, the ineffectiveness of all of the cuing treatments studied strongly suggests an optimal functioning of the retrieval processes.

In all, this series of experiments indicates a timedependent retrieval variability that runs parallel with the time-dependent retention-performance level.

The main finding of these experiments is the demonstration of time-dependent cuing effectiveness for three of the cuing treatments studied. The last experiment indicates that retention performance can be significantly enhanced by a pretest exposure to some training events $1 \mathrm{~h}$ as well as 21 days after initial training. However, the nature of the effective retrieval cues changes according to the length of the retention interval. This experiment attests to the reliability of the results obtained in the first series of experiments. Hence, the nine cuing treatments studied can be divided on the basis of their effectiveness on cued animals relative to noncued animals.

Roughly, four main categories can be proposed (see Figure 7): (1) treatment always ineffective, such as Control Treatment B, or (2) treatment always effective when retention performance is depressed, such as Treatments BG, BGS, and TRIAL. This suggests that attributes related to the UCS remain persistently of importance in the memory trace and that their reactivation improves retrieval processes when disrupted, independent of the length of the TTI.

The other two categories concern treatments whose effectiveness clearly depends on the duration of the retention interval, as confirmed by the second experiment. (3) The effectiveness of Treatment BL has been shown to decrease over time. It can be proposed that shortly after training, memory attributes are well individualized. At that time, exposure to specific events, such as the CS, would be highly effective in promoting retrieval, espe-
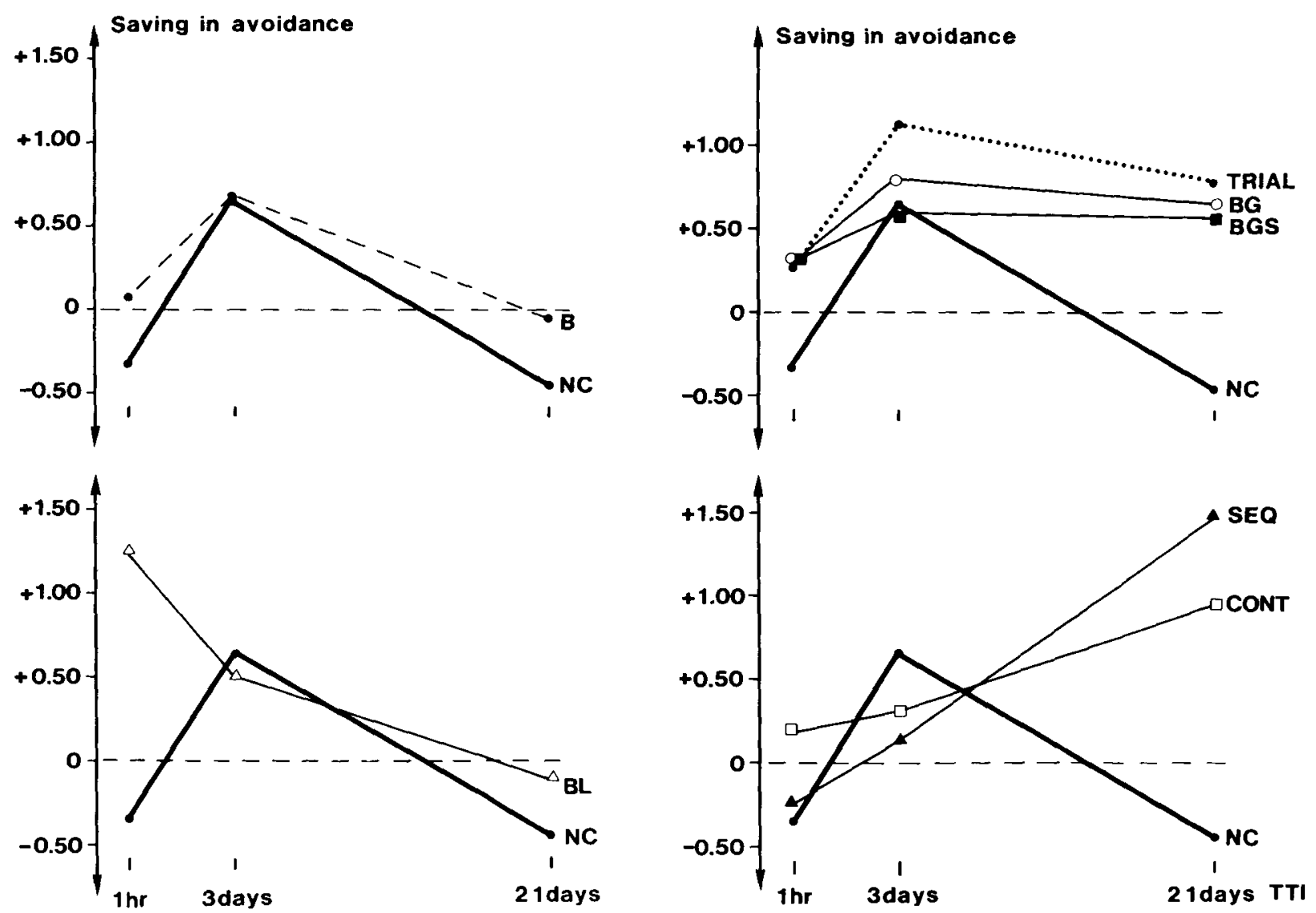

Figure 7. Summary of the evolution of retention performance for the noncued animals (NC) and the cued animals suggested by the first three experiments. Treatment B was never effective; Treatments BG, BGS, and TRIAL were effective when retention performance was poor. The effectiveness of Treatment BL decreased, whereas the effectiveness of Treatments CONT and TRIAL increased over time. The differential effectiveness obtained for those three treatments was confirmed in the last experiment. 
cially because of the strengthened connections between CS and UCS attributes, closely tied with the response. After longer intervals, this treatment may lose its effectiveness because of a memory loss for specific attributes of the CS (for more details, see Gisquet-Verrier \& Alexinsky, 1989). (4) At the opposite extreme, the effectiveness of an exposure to the well-ordered sequence of events (Treatment SEQ) or to the experimental context (Treatment CONT) increases over time. The effectiveness of treatments involving several related training events long after training, that is, when the memory trace has become more abstract and schematized (Squire \& Cohen, 1984), suggests that, at that time, the reactivation of the relationships between the memory attributes is at least as important as the reactivation of the attributes themselves for enhancing the retrieval of the training episode. This latter interpretation seems to preclude the well-accepted notion that, to be effective, information delivered during cuing must match the information presented at the time of storage (Spear, 1978). It seems more likely that matching has to occur between the information contained in the cuing treatment and the internal representation that is present at the time of testing. In all, the differential effectiveness of a variety of cuing treatments demonstrates that there is a change in the nature of the "key attributes" able to trigger the reactivation of the whole memory trace. This, in turn, strongly suggests that the structure of the memory trace is modified as a function of the length of the retention interval.

\section{REFERENCES}

Anisman, H. (1975). Time-dependent variations in aversively motivated behaviors: Nonassociative effects of cholinergic and catecholaminergic activity. Psychological Review, 82, 359-385.

Balaz, M. A., Gustin, P., Cacheiro, H., Miller, R. R. (1982). Blocking as retrieval failure: Reactivation of associations to a blocked stimulus. Quarterly Journal of Experimental Psychology, 34B, 99-113.

BowER, G. H. (1967). A multi-component theory of the memory trace. In J. W. Spence \& J. T. Spence (Eds.), The psychology of learning and motivation (Vol. 1). New York: Academic Press.

BRush, F. R. (1971). Retention of aversively motivated behavior. In F. R. Brush (Ed.), Aversive conditioning and learning. New York: Academic Press.

Cherkin, A. (1971). Biphasic time course of performance after onetrial avoidance training in the chick. Behavioral Biology, 5, 379-381.

DEVIETT, T. L., HopFer, T. M. (1974). Complete amnesia induced by ECS and complete recovery of memory following reinstatement treatment. Physiology \& Behavior, 12, 599-603.

DEWEER, B. (1986). Pretest cuing after forgetting of a food-motivated maze task in rats: Synergistic action of context and reinforcement. Animal Learning \& Behavior, 14, 249-256.

Deweer, B., Sara, S. J., Hars, B. (1980). Contextual cues and memory retrieval in rats: Alleviation of forgetting by a pretest exposure to background stimuli. Animal Learning \& Behavior, 8, 265-272.

Gatti, S. V., Pais, N., \& Weeks, J. R., JR. (1975). Effect of reinstatement procedures on retention of differential appetitive responding. Bulletin of the Psychonomic Society, 6, 57-60.

GISQUET-VERRIER, P., \& AleXINSKY, T. (1986). Does contextual change determine long-term forgetting? Animal Learning \& Behavior, 14. 349-358.

Gisquet-Verrier, P., * Alexinsky, T. (1988). Time-dependent fluctuations of retention performance in an aversively motivated task Animal Learning \& Behavior, 16, 58-66.

Gisquet-Verrier, P., \& Alexinsky, T. (1989). Facilitative effect of a pretest exposure to the CS: Analysis and implications for the memory trace. Manuscript submitted for publication.

Gold, P. E., KING, R. D. (1974). Storage failure versus retrieval failure. Psychological Review, 81, 465-469.

Gordon, W. C., \& Mowrer, R. R. (1980). An extinction trial as a reminder treatment following electroconvulsive shock. Animal Learning \& Behavior, 8, 363-367.

Gordon, W. C., Smith, G. J., Katz, D. S. (1979). Dual effects of response blocking following avoidance learning. Behavior Research \& Therapy, 17, 479-487.

Hendersen, R. W., Patterson, J. M., \& Jackson, R. L. (1980). Acquisition and retention of instrumental behavior by a cue-signaling air blast: How specific are conditioned anticipations? Leaming \& Motivation, 11, 407-426.

Holloway, F. A., \& Wansley, R. A. (1973). Multiple retention deficits at periodic intervals after passive avoidance learning. Science, 80 , 208-210.

HUPPERT, F. A., \& DeUtsCh, J. A. (1969). Improvement on memory with time. Quarterly Joumal of Experimental Psychology, 21, 267-271.

KAMIN, L. J. (1957). The retention of an incompletely learned avoidance response. Journal of Comparative \& Physiological Psychology, 50, $457-460$.

Kasprow, W. J., Cacheiro, H., Balaz, M. A., \& Miller, R. R. (1982). Reminder-induced recovery of associations to an overshadowed stimulus. Learning \& Motivation, 13, 155-166.

KLEIN, S. B. (1972). Adrenal-pituitary influence in reactivation of avoidance-learning memory in the ral after intermediate interval. Journal of Comparative \& Physiological Psychology, 79, 341-359.

KLEIN, S. B., \& SPEAR, N. E. (1970). Reactivation of avoidance-learning memory in the rat after intermediate retention intervals. Journal of Comparative \& Physiological Psychology, 72, 498-504.

KoppenaAl, R. J., JAgoda, E., Cruce, J. A. F. (1967). Recovery from ECS-produced amnesia following a reminder. Psychonomic Science, 9, 293-294.

LEWIS, D. J. (1979). Psychobiology of active and inactive memory. Psychological Bulletin, 86, 1054-1083.

MacArdy, E., Kasenow, P. M., Hopkins, J., Ruccio, D. C. (1987, May). Changes in the effectiveness of a non-contingent footshock as a function of retention interval. Paper presented at the meeting of the Midwestern Psychological Association, Chicago, IL.

Messenger, J. B. (1971). Two stages recovery in Sepia. Nature, 232, 202-203.

Miller, R. R., Kasprow, W. J., Schachtman, T. R. (1986). Retrieval variability: Sources and consequences. American Journal of Psychology, 99, 145-218.

MILLER, R. R., \&PRINGER, A. D. (1972). Induced recovery of memory in rats following electroconvulsive shock. Physiology \& Behavior, 8, 645-651.

Misanin, J. R., Miller, R. R., \& LeWIs, D. J. (1968). Retrograde amnesia produced by electroconvulsive shock after reactivation of a consolidated memory trace. Science, 160, 554-555.

Rohrbaugh, M., Riccio, D. C. (1970). Paradoxical enhancement of learned fear. Journal of Abnormal Psychology, 75, 210-216.

Rohrbaugh, M., Riccio, D. C., \& Arthur, A. (1972). Paradoxical enhancement of conditioned suppression. Research \& Therapy, 10, 125-130.

SANDERS, G. D., BARLow, J. J. (1971). Variation in retention performance during long term formation. Nature, 232, 203-204.

SARA, S. J. (1973). Progressive development of avoidance response after training, ECS, and repeated testing. Bulletin of the Psychonomic Society, 2, 134-136. 
Silvestri, R., Rohrbaugh, M., \& Riccio, D. C. (1970). Conditions influencing the retention of learned fear in young rats. Developmental Psychology, 2, 380-395.

SPEAR, N. E. (1973). Retrieval of memory in animals. Physiological Review, 80, 1163-1194.

SPEAR, N. E. (1978). The processing of memories: Forgetting and retention. Hillsdale, NJ: Erlbaum.

Spear, N. E., Hamberg, J. M., \& Bryan, R. (1980). Forgetting of recently acquired or recently reactivated memories. Leaming \& Motivation, 11, 456-475.

SPEAR, N. E., \& Parsons, P. (1976). Analysis of a reactivation treatment: Ontogeny and alleviated forgetting. In R. Davies \& W. Roberts (Eds.), Coding processes in animal memory (pp. 135-165). Hillsdale, NJ: Erlbaum.

SQuire, L. R., \& CoHEN, N. J. (1984). Human memory and amnesia.
In L. Lynch, J. L. McGaugh, \& N. M. Weinberger (Eds.), Neurobiology of learning and memory (pp. 3-64). New York: Guilford.

Thomas, D. A., Riccio, D. C. (1979). Forgetting of a CS attribute in a conditioned suppression paradigm. Animal Learning \& Behavior, 7, 191-195.

Tulving, E., Thomson, D. M. (1973). Encoding specificity and retrieval processes in episodic memory. Psychological Review, 80, 352-373.

Tulving, E., \& Watkins, M. J. (1975). Structure of memory traces. Psychological Review, 82, 261-275.

UNDERWOOD, B. J. (1969). Attributes of memory. Psychological Review, 76, 559-573.

(Manuscript received June 28, 1988; revision accepted for publication February 5, 1989.) 